\title{
Robinson dall'avventura al mito. «Robinsonnades»e generi affini, a cura di Maria Chiara Gnocchi e Carmelina Imbroscio
}

\section{Francesca Torchi}

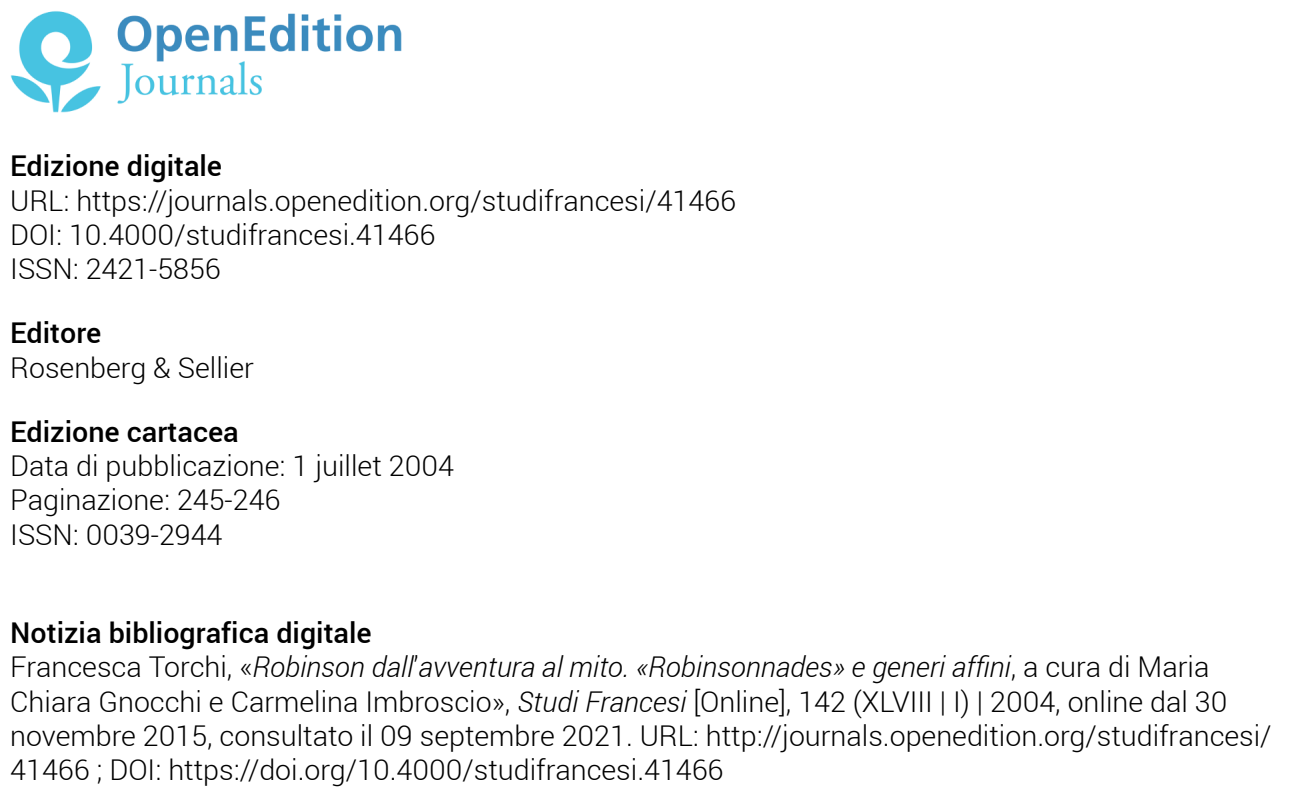

Questo documento è stato generato automaticamente il 9 septembre 2021.

\section{(ब) $\odot \Theta$}

Studi Francesi è distribuita con Licenza Creative Commons Attribuzione - Non commerciale - Non opere derivate 4.0 Internazionale. 


\title{
Robinson dall'avventura al mito. «Robinsonnades» e generi affini, a cura di Maria Chiara Gnocchi e Carmelina Imbroscio
}

\author{
Francesca Torchi
}

\section{NOTIZIA}

AA.VV., Robinson dall'avventura al mito. «Robinsonnades» e generi affini, a cura di Maria Chiara GNocCHI e Carmelina IMBRoscio, Atti del Convegno di Bologna (12-13 novembre 1999), Bologna, CLUEB, «Heuresis Strumenti», 2000, pp. 271.

1 Robinson Crusoe non è morto. L'impressionante quantità di opere - per voler rimanere solo in ambito letterario - che hanno riprodotto, rivisitato, trasformato e attualizzato la storia del suo naufragio e della sua permanenza sull'isola deserta, testimonia che il personaggio creato da Daniel Defoe agli inizi del Settecento è più vivo che mai... Molti degli aspetti costitutivi della sua storia originaria sono sopravvissuti trasmigrando di opera in opera sino ai giorni nostri, divenendo i tasselli di un archetipo universale. L'avventura di Robinson ha così assunto i caratteri del mito. E chi dice mito, dice sogno eterno.

2 Robinson dall'avventura al mito. "Robinsonnades» e generi affini raccoglie gli Atti di un convegno che si è tenuto nel novembre 1999, che ha riunito i contributi di molti specialisti di letteratura italiani e stranieri, su alcuni aspetti delle riscritture del Robinson Crusoe. Pur essendo gli interventi proposti di carattere vario, risulta evidente un percorso evolutivo della «robinsonnade» come genere. Già nel Settecento il rornanzo di Defoe conosce un enorme successo ed è citato e imitato. L'Ottocento vede un moltiplicarsi delle versioni del Robinson a scopo pedagogico o nell'intento di assecondare una vera e propria moda legata all'imrnaginario esotico; e le sue riscritture 
rispecchiano soprattutto l'affermarsi di un modello letterario estetico e morale, da rivisitare e da cui prendere o meno le distanze. È proprio nel Novecento, invece, che la figura di Robinson diventa mito. La storia originaria perde progressivamente consistenza e si spoglia dei suoi significati primari. Il mito è ciò che rimane: un'essenziale rete di topoi che permette a Robinson, a Venerdì, all'isola deserta di prendere vita in più letterature rivestendo sempre ruoli nuovi legati ai bisogni profondi di quell'epoca o di quella società.

3 Dei diciotto saggi contenuti nel volume, ben otto riguardano letterature di espessione francese. Questo puo far intuire il fascino che la storia di Robinson ha suscitato negli scrittori francesi sino ai giorni nostri e come per essi la percezione di questa storia come mito abbia costituito un palinsesto su cui 'scrivere' il proprio tempo. Carmelina Imbroscio mostra come ne L'île inconnue, ou Mémoires du Chevalier de Gastine (1783-1787) di Guillaume Grivel, il mito di Robinson venga a coincidere con il mito dell'età dell'oro, della fondazione di una civiltà utopica che, a differenza di quel che accade nel romanzo di Defoe, si sviluppa sulla negazione della civiltà di partenza. Carla Pellandra presenta le robinsonnades della Comtesse de Ségur, scrittrice ottocentesca per l'infanzia, tutt'oggi molto nota. La Contessa, con spirito antibritannico e sciovinista, fa delle sue microstorie un'esaltazione della colonizzazione francese, afferrnando la superiorità dei naufragi e dipingendo gli abitanti dell'isola veri bons sauvages, mansueti, buffi, ma irrimediabilmente inferiori. Ne «I Robinson francesi del secondo Ottocento» Nadia Minerva osserva i mutamenti della fortuna di Robinson nelle numerosissime opere che propongono una variante del testo eponimo ed evidenzia gli intenti pedagogici di questi testi che rimangono troppo figli del proprio tempo e della mentalità che li ha prodotti. Si distinguono però, tra le varie rivisitazioni, le robinsonnades di Jules Verne, su cui si sofferma Piero Gondolo della Riva. I romanzi esaminati denotano una vera passione dell'autore per il tema del naufragio e per l'azione che si svolge in uno spazio chiuso e circoscritto (l'isola, la grotta, ma anche, ad esempio, il sottomarino di Vingt mille lieues sous les mers), oltre ad una continua necessità di introdurre spunti e situazioni sempre nuovi e ingegnosi, caratteristica comunque di tutta la sua opera.

4 Carminella Biondi, nel saggio "L'Arbre du voyageur di Bruno Bontempelli. La fine del mito?» propone la lettura di questo Robinson novecentsco in cui è messa in scena la quête di un cavaliere senza speranze. Non solo il percorso iniziatico del protagonista non si concluderà, ma l'isola, simbolo di una nuova occasione per rinascere, non verrà mai raggiunta. Ci si trova così di fronte ad una serie di riferimenti ai Robinson di Defoe, di Tournier e ai terni classici dell'iniziazione come il labirinto o la discesa agli inferi in un romanzo in cui il finale segna però la possibile morte di un mito. Tra gli autori francesi che hanno contribuito alla rivisitazione del Robinson Crusoe vengono ricordati inoltre Saint-John Perse, Valéry, Giraudoux, Hervé e soprattutto Michel Tournier con il suo Vendredi ou les limbes du Pacifique, di cui Arlette Bouloumié offre un'interpretazione in chiave antropologica. Tra gli autori di espressione francese figurano il quebecchese Poulin (Gilles Dupuis) e il belga Compère (Elena Pessini), che nel suo Robinson 86 dichiara la propria dipendenza sia dal Robinson di Defoe che da quello di Tournier per prendere poi da entrambi le distanze: il percorso iniziatico del suo eroe non sfocerà in una situazione di equilibrio e di felicità finale.

5 Il volume presenta inoltre saggi che - prendendo in considerazione l'eredità di Robinson a partire dal Settecento, in ambito tedesco (Carla Consolini), spagnolo (Maurizio Fabbri), inglese (Gabriella Morisco) - ne percorrono la fortuna ottocentesca 
non solo in Francia ma anche in Italia (Claudio Gallo). L'ultima parte del volume è invece dedicata al Novecento, secolo della rivisitazione più radicale della robinsonnade. La storia di Robinson prende ad esempio nuova forma tra le Alpi austriache (Rita Svandrlik), e l'isola come simbolo dell'universo chiuso si sposta geograficamente tre le montagne. Gabriella Imposti offre un panorama dei Robinson nella letteratura russa contemporanea, mentre Roberto Vecchi propone una lettura di A cidade e a serras del portoghese Eça de Queirós, in cui il naufragio diviene una delle possibili metafore distintive della modernità. Il Novecento è inoltre il periodo in cui le riscritture della storia di Robinson diventano, secondo un'ottica esistenzialista, il luogo narrativo in cui trovano espressione le questioni della ricerca identitaria e della relazione con l'Altro, fondamentali per le letterature postcoloniali. Silvia Albertazzi mostra, nel suo saggio dedicato a Foe di Coetzee, come per un autore anglofono sudafricano subire il fascino del mito che incarna il canone occidentale dell'epoca della colonizzzione significhi necessariarnente affrontare il problema della creazione di un nuovo canone in grado di esprimere la propria identità.

Aprono e chiudono la raccolta due saggi di carattere più generale che contribuiscono a dare organicità all'insieme dei contributi. Il primo è quello di Mario Domenichelli, «Robinson e robinsonnades: la tempesta e altri paradigmi», in cui la figura mitica del Robinson di Defoe, protomodello della cultura colonial-imperialista, viene messa a confronto con l'Ulisse dantesco mito della libertà individuale, e con Noè, capostipite eletto di una società migliore, utopica. In quest'ottica l'autore propone una lettura degli elementi dei romanzo di Defoe, come ad esempio la tempesla o la figura di Venerdì, legata ai motivi sociali, morali ed economici dell'epoca. Anche l'intervento di Ettore Finazzi-Agrò, che chiude il volume, offre una lettura trasversale di robinsonnades inglesi, francesi, quebecchesi, portoghesi, austriache, ecc. dedicandosi allo studio della figura del cannibale nell'immaginario europeo per il quale l'isola è spazio utopico e atopico in cui tutto è possibile. 